\title{
The Effect of the Fractional Carbon Dioxide Laser on Improving Minoxidil Delivery for the Treatment of Androgenetic Alopecia
}

\author{
Manal Salah ${ }^{1}$, Nevien Samy ${ }^{1 *}$, Marwa Mohamed Fawzy² ${ }^{2}$ Abdel Razik Farrag ${ }^{3}$, Hany Shehata ${ }^{4}$, Aya Hany ${ }^{4}$ \\ ${ }^{1}$ Department of Medical Applications of Laser, National Institute of Laser Enhanced Sciences (N.I.L.E.S), Cairo University, \\ Cairo, Egypt \\ ${ }^{2}$ Department of Dermatology, Kasr Al Ainy Hospital, Cairo University, Cairo, Egypt \\ ${ }^{3}$ Pathology Department, Medical Division Research National Research Centre, Cairo, Egypt, \\ ${ }^{4}$ Department of Dermatology and Venereology, National Research Centre (NRC), Cairo, Egypt
}

*Correspondence to
Nevien Samy
Tel: $+201005755739 ;$
Email:
nevien_a_sami@hotmail.com

Published online January 18, 2020

\begin{abstract}
Introduction: Recently, laser treatment for hair loss has become very popular. Laser-assisted drug delivery (LAD) is an evolving technology with potentially broad clinical applications. This work aims at inspecting the effect of the fractional carbon dioxide laser $\left(\mathrm{CO}_{2}\right)$ on improving the delivery of minoxidil in patients with androgenetic alopecia and detecting the role of the fractional $\mathrm{CO}_{2}$ laser in its treatment.

Methods: We enrolled 45 Egyptians with male androgenetic alopecia (MAGA); clinical grading was assessed based on Norwood-Hamilton classification. The patients were divided into 3 groups: the first group (combined group) received the fractional $\mathrm{CO}_{2}$ laser session followed by topical application of minoxidil and also in between sessions; the second group received fractional $\mathrm{CO}_{2}$ laser sessions only and 6 sessions with 2-week intervals were performed; the third group applied topical minoxidil only for 3 months. Global photographs and dermoscopic assessments were performed before treatment and 3 months after the treatment.

Results: Several dermoscopic findings were detected, including peripilar sign, hair diversity, yellow spot, white dots, and arborizing red lines. The number of double hair units significantly increased after the treatment in the combined group. The mean number of hair after the treatment in the 3 groups significantly increased, mostly in the combined group. The hair thickness (thin \& thick) significantly increased after the treatment in the combined group and the fractional group; however, in the minoxidil group, only thin hair thickness increased. In all the 3 groups, there was a significant improvement in hair count and thickness.

Conclusion: The ablative fractional $\mathrm{CO}_{2}$ laser alone or combined with minoxidil may serve as an additional treatment for MAGA.

Keywords: Male androgenetic alopecia; Ablative fractional $\mathrm{CO}_{2}$ laser; Minoxidil; Laser-assisted drug delivery.
\end{abstract}

\section{Introduction}

Androgenetic alopecia (AGA) (also known as androgenic alopecia or alopecia androgenetica) is a loss of hair which happens as a result of prime susceptivity of hair follicles to androgenic miniaturization. It is the most popular reason for hair loss affecting up to $70 \%$ of males and $40 \%$ of females at some time in their lives. Men classically present with the recession of hairline at the temples and vertex balding, while women usually diffusely thin over the top of their scalps with preserving the frontal hairline. ${ }^{1}$

Various treatment modalities such as various drug therapies, surgical hair transplantation, and laser treatment have been used for the treatment of AGA. ${ }^{1}$
Drugs approved by the Food and Drug Administration (FDA) include minoxidil and finasteride. Topical minoxidil is commonly used in the treatment of AGA; it prolongs the anagen growth phase and gradually develops miniaturized follicles (vellus hairs) into mature terminal hairs. A course of 3-6 months of treatment is required to decrease hair fall and 6-12 months to improve scalp coverage. The continuation of the treatment is required to keep the profit. $^{2}$

Recently, laser treatment for hair loss has become very popular; it has also been promoted as a preventative measure against AGA as there is evidence that laser light stimulates hair growth at some wavelengths. ${ }^{3}$ 
Laser-assisted drug delivery (LAD) is an evolving technology with potentially broad clinical applications. Lasers stimulate drug delivery by means of 3 processes; 1) tissue ablation, which removes the stratum corneum and the most superficial layers of the epidermis; 2) photomechanical waves, resulting from the conversion of light into mechanical energy giving the laser a therapeutic effect; and 3) non-ablative resurfacing where thermal and physical injuries disrupt the skin barrier, promoting the delivery of medications through these laser channels. ${ }^{4}$

Fractional laser therapy has been introduced to treat AGA as it is a treatment for alopecia and can also prevent hair loss recurrence. ${ }^{5}$

Fractional lasers can stimulate hair growth when restricted to appropriate settings. Although the precise mechanisms of specific fractional lasers on hair regrowth are not well interpreted yet, it is most likely due to trauma stimulated wound healing. Hair follicle stem cells supply descendants which travel to the epidermal defect and promote re-epithelialization. The process of cutaneous wound healing increases significantly during the anagen phase, and more hair follicles regrow after tissue injury. ${ }^{6}$

In the present study, we aimed to inspect the effect of the fractional carbon dioxide laser on improving the delivery of minoxidil in MAGA male patients and detect the role of fractional $\mathrm{CO}_{2}$ laser alone in the treatment.

\section{Patients and Methods}

Patients

In this open labeled clinical study, we enrolled 45 Egyptians with male AGA (MAGA) between 21 and 45 years of age. The study was performed at the Laser Unit in the National Research Center (NRC). All patients provided written informed consent.

The diagnosis was based on a thorough physical examination and detailed history taking. The clinical grading was assessed based on the Norwood-Hamilton classification. $^{7}$

We excluded patients having a history of systemic disease in the past 6 months, those on treatment with drugs that could affect the hair cycle during the past 3 months, photosensitive patients, and those who had undergone surgery for alopecia (e.g. hair transplantation). It was not allowed to use any other topical agents or to take any medication throughout the study period.

\section{Treatment Protocol}

The patients were divided into 3 groups as follows: the first group (combined group) received fractional $\mathrm{CO}_{2}$ laser sessions followed by immediate topical application of 6 puffs $(=1 \mathrm{~mL})$ of minoxidil 5\% solution twice daily (Performa Extra Strength $®$, Egypt) and also in between the sessions; the second group received fractional $\mathrm{CO}_{2}$ laser sessions only and 6 sessions with 2 -week intervals were performed; and the third group applied topical minoxidil only for 3 months. The fractional $\mathrm{CO}_{2}$ laser
(DEKA Smartxide ${ }^{2}$ DOT/RF c60, Italy) with the following parameters was used: DOT fractional scanning mode, one pulse, spot size $15 \mathrm{~mm}$, power $5 \mathrm{~W}$, dwell time $500 \mu \mathrm{s}$, smart stack 3, emission mode Deka pulse (DP), spacing $700 \mu \mathrm{m}$, density $8.7 \%$, fluence $4.68 \mathrm{~J} / \mathrm{cm} 2$, pulse energy $51.6 \mathrm{~mJ}$, and scan mode smart track.

In each patient, a well-demarcated area of $25 \mathrm{~mm}$ diameter was identified to apply the laser in the right and left parietal areas and the vertex of hair. The treatment procedure did not require the use of topical anesthetics. At the end of the treatment, the patients were asked to fill in a questionnaire to indicate their satisfaction with the overall treatment outcomes and mention any noticed side effects. They were also asked to assess the treatment outcome using a 7-point global-assessment scale $^{3}$ as follows: significant deterioration, -3 points; moderate deterioration, -2 points; slight deterioration, -1 point; no change, 0 point; slight improvement, +1 point; moderate improvement, +2 points; and significant improvement, +3 points. Global photographs and dermoscopy assessments were done before starting the treatment and 3 months later.

\section{Pre/Post-operative Care}

Before the session, the patients were instructed to clean their heads and not to apply anything on it. After the session, the patients were instructed to avoid direct sun exposure or wear a head cap.

\section{Outcome Evaluation}

\section{Global Photo Assessment}

Global photographs (taken at the baseline and after 3 months) were evaluated independently by 2 dermatologists, who were blinded to the study protocol. The photographs were scored using the 7-point globalassessment scale ${ }^{3}$ as mentioned before.

\section{Dermoscopic Assessment}

Dermoscopic examinations of areas of hair loss on the patients' scalps were carried out using a DermLite II Pro dermoscopy (3Gen, San Juan Capistrano, CA, USA) which provides 10 -fold magnification. We photographed the dermoscopic images using a Nikon Coolpix 4500 digital camera (Nikon, Tokyo, Japan) attached to a DermLite II Pro dermoscopy. Photos were assessed by a dermatologist for the determination of the following dermoscopic features:

(1) Peripilar sign: This is a brown halo, about $1 \mathrm{~mm}$ in diameter, surrounding the emergence of the hair shaft which is pathologically correlated to perifollicular inflammation. It was assumed that this sign is a result of post-inflammatory perifollicular pigmentation. ${ }^{8}$

(2) Hair diameter diversity (HDD): This is variability in the diameter of hair shafts, which signifies that vellus hairs represent more than $20 \%$ of all the hairs in the same view. ${ }^{8}$ 
(3) Multiple hair units: The number of hairs in one pilosebaceous unit ranges from one to 3 in healthy persons. Sometimes, a 4 -hair unit can be seen. ${ }^{9}$

(4) Yellow spots which represent hair follicle ostia devoid of any hairs (empty hair follicles). ${ }^{10}$

(5) White dots regularly scattered between the follicular ostia with a diameter of $0.2-0.3 \mathrm{~mm}$. By histopathology, it was shown they correspond to the empty hair follicle ostia or to the epidermal part of eccrine sweat ducts. ${ }^{11}$

(6) Structureless red areas corresponding pathologically to subpapillary vascular plexus. ${ }^{11}$

The morphometric analysis was performed at the Pathology Department, National Research Center using the Leica Qwin 500 Image Analyzer (LEICA Imaging Systems Ltd, Cambridge, England), which consists of the Leica DM-LB microscope with the JVC color video camera connected to a computer system Leica Q 500IW (Figure 1).

The morphometric analysis was performed on the photos of each case. We started counting the number of hair (thick and thin). Then we measured the width of the hair by drawing a line from one surface until the opposite surface by using the interactive measurement software of the system. The outcome appears automatically on the monitor in the form of the number or thickness measured in $\mu \mathrm{m}$.

\section{Statistical Methods}

Statistical analysis was performed using IBM $^{\odot}$ SPSS $^{\odot}$ Statistics version 22 (IBM ${ }^{\odot}$ Corp., Armonk, NY, USA). Numerical data were expressed as mean and standard deviation or median and range as appropriate. Qualitative data were expressed as frequency and percentage. The chi-square test or Fisher exact test was used to examine the relation between qualitative variables. For not normally distributed quantitative data, the comparison between 3 groups was done using the Kruskal-Wallis test (non-parametric ANOVA), and then the post hoc test was used for pair-wise comparison based on KruskalWallis distribution. The Wilcoxon signed-rank test (non-parametric paired $t$ test) was used to compare 2

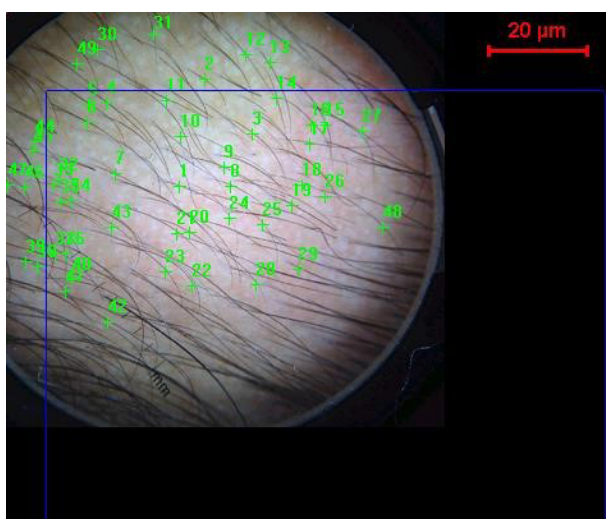

Figure 1. The Dermoscopic Photo as Seen by the Image Analyzer. consecutive measures of numerical variables. Due to multiple comparisons, the $P$ value was corrected using the Bonferroni method. All tests were two-tailed. A $P$ value < 0.05 was considered significant.

\section{Results}

General Information

This study included 45 male patients whose ages ranged from 21 to 45 years with a mean of $31.48 \pm 6.62$ SD. All patients had a positive family history. The NorwoodHamilton stages of hair loss were detected as follows: stage II, 3 participants; stage III, 27 participants; stage IV, 2 participants; stage V, 7 participants; and stage VI, 6 participants. The duration of the disease ranged from 6 months to 30 years with a median of 7 years.

\section{Dermoscopic Findings}

(1) Peripilar sign: There was no significant difference $(P=0.089)$ between the groups regarding the peripilar sign before treatment. After treatment, there is a significant difference $(P=0.036)$ between the 3 groups where it is more present in the fractional group than the other 2 groups as it represented $86.7 \%$ while in the other 2 groups it represented only $46.7 \%$ (Table 1 and Figure 2).

(2) Hair diameter diversity (HDD): There was no significant difference between the 3 groups regarding hair diversity before and after treatment. However, it was noticed that the percentage decreased after treatment in
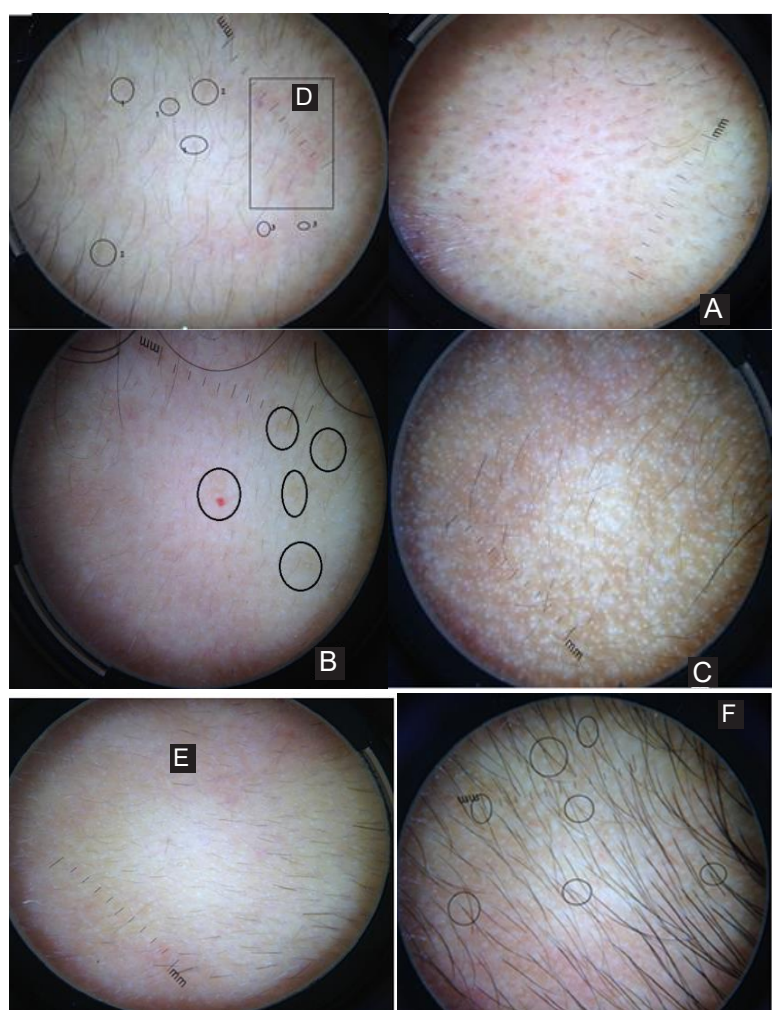

Figure 2. Dermoscopic Findings. (A) Peripilar sign. (B) Yellow spot (C) White dots. (D) Structureless red areas. (E) Hair diversity. (F) Multifollicular hair units (MFHU). 
Table 1. Dermoscopy Assessment of Peripilar Sign

\begin{tabular}{lccccc}
\hline & & $\begin{array}{c}\text { Combined } \\
(\mathbf{n = 1 5})\end{array}$ & $\begin{array}{c}\text { Fractional } \\
(\mathbf{n = 1 5})\end{array}$ & $\begin{array}{c}\text { Minoxidil } \\
(\mathbf{n = 1 5})\end{array}$ & $\begin{array}{c}\text { Total } \\
(\mathbf{n = 4 5})\end{array}$ \\
\hline \multirow{2}{*}{ Before } & $<30 \%$ & $7(46.7 \%)$ & $2(13.3 \%)$ & $7(46.7 \%)$ & $16(35.6 \%)$ \\
& $>30 \%$ & $8(53.3 \%)$ & $13(86.7 \%)$ & $8(53.3 \%)$ & $29(64.4 \%)$ \\
\multirow{2}{*}{ After } & $<30 \%$ & $8(53.3 \%)$ & $2(13.3 \%)$ & $8(53.3 \%)$ & $18(40.0 \%)$ \\
& $>30 \%$ & $7(46.7 \%)$ & $13(86.7 \%)$ & $7(46.7 \%)$ & $27(60.0 \%)$ \\
\hline
\end{tabular}

a $P$ value $<0.05$ is statistically significant.

Table 2. Dermoscopy Assessment of Hair Diameter Diversity

\begin{tabular}{|c|c|c|c|c|c|c|}
\hline & & $\begin{array}{c}\text { Combined } \\
(n=15)\end{array}$ & $\begin{array}{c}\text { Fractional } \\
(\mathrm{n}=15)\end{array}$ & $\begin{array}{c}\text { Minoxidil } \\
(n=15)\end{array}$ & $\begin{array}{c}\text { Total } \\
(n=45)\end{array}$ & $P$ Value \\
\hline \multirow{2}{*}{ Before } & $<20 \%$ & $3(20.0 \%)$ & $9(60.0 \%)$ & $5(33.3 \%)$ & $17(37.8 \%)$ & \multirow{2}{*}{0.071} \\
\hline & $>20 \%$ & $12(80.0 \%)$ & $6(40.0 \%)$ & $10(66.7 \%)$ & $28(62.2 \%)$ & \\
\hline \multirow{2}{*}{ After } & $<20 \%$ & $9(60.0 \%)$ & $8(53.3 \%)$ & $10(66.7 \%)$ & $27(60.0 \%)$ & \multirow{2}{*}{0.757} \\
\hline & $>20 \%$ & $6(40.0 \%)$ & $7(46.7 \%)$ & $5(33.3 \%)$ & $18(40.0 \%)$ & \\
\hline
\end{tabular}

the combined and minoxidil groups (Table 2).

(3) Multiple hair units

(a) Double hair units: There was no significant difference between the 3 groups before $(P=0.056)$ and after $(P=0.091)$ treatment. There was a significant increase in the number of double hair units before and after treatment in the combined patients' group $(P=0.012)$.

(b) Triple hair units: There was no significant difference between the 3 groups before $(P=0.143)$ and after $(P=0.325)$ treatment. There was no significant difference within each group before and after treatment. However, it was noticed that the maximum number of triple units $(\mathrm{n}=3)$ was in the combined group after treatment.

(4) Yellow spots (5) White dots (6) Structureless red areas: The last 3 findings were present in all patients before and after treatment.

\section{Dermoscopical Notices}

- The areas of post-laser hyperpigmentation which showed new hair growth.

- It was also noticed that newly grown hair was thick dark hair.

- Light-colored/white hair was dermoscopically difficult to assess.

Image Analysis Findings

(1) Hair count

(a) Total hair count

There was a significant difference between the 3 groups before $(P=0.061)$ and after $(P=0.578)$ treatment. There was a significant increase in the mean number of hair after treatment in the 3 groups, where it was most significant in the combined patient group $(P=0.001)$, then in the fractional group $(P=0.005)$, and then in the minoxidil group $(P=0.007)$.

\section{(b) Thick Hair Count}

There was no significant difference between the 3 groups before $(P=0.593)$ and after $(P=0.911)$ treatment. There was a significant increase in the count of thick hair after treatment in all the groups, where it was most significant in the combined patient group $(P=0.001)$ and the fractional group $(P=0.001)$ and then the minoxidil group $(P=0.009)$.

\section{(c) Thin to Medium Hair Count}

There was a significant difference between the 3 groups before treatment $(P=0.035)$. There was a significant increase in the count of thin hair after treatment in the combined group $(P=0.001)$ and the fractional group $(P=0.006)$, but not in the minoxidil group $(P=0.094)$. However, there was no significant difference between the 3 groups after treatment $(P=0.345)$ (Table 3$)$.

\section{(2) Hair Thickness \\ (a) Thick Hair Thickness}

There was no significant difference between the 3 groups before $(P=0.470)$ and after $(P=0.138)$ treatment. There was a significant increase in the thickness of thick hair after treatment in the fractional group $(P=0.008)$ and the combined group $(P=0.042)$. There was no significant increase in the thickness of thick hair after treatment in the minoxidil group $(P=0.110)$.

\section{(b) Thin to Medium Hair Thickness}

There was a significant difference between the 3 groups before treatment $(P=0.035)$. There was a significant increase in the thickness of thin hair after treatment in the 3 groups; the combined group $(P=0.001)$, the fractional group $(P=0.001)$, and the minoxidil group $(P=0.001)$. However, there was no significant difference between the 3 groups after treatment $(P=0.0345)$ (Table 4 and Figure 3 ). 
Table 3. Dermoscopy Assessment of the Hair Count

\begin{tabular}{|c|c|c|c|c|c|}
\hline Mean Number of Hair Units & & $\begin{array}{c}\text { Combined } \\
(n=15)\end{array}$ & $\begin{array}{l}\text { Fractional } \\
\quad(n=15)\end{array}$ & $\begin{array}{l}\text { Minoxidil } \\
(n=15)\end{array}$ & $P$ Value \\
\hline \multirow{3}{*}{ Total } & Before & 20.2 & 35.3 & 32.7 & 0.061 \\
\hline & After & 41.0 & 56.5 & 50.1 & \multirow{2}{*}{0.578} \\
\hline & $P$ value & $0.001^{\mathrm{a}}$ & $0.005^{\mathrm{a}}$ & $0.007^{\mathrm{a}}$ & \\
\hline \multirow{3}{*}{ Thick hair } & Before & 2.9 & 3.1 & 4.7 & 0.593 \\
\hline & After & 10.3 & 9.2 & 14.2 & \multirow{2}{*}{0.911} \\
\hline & $P$ value & $0.001^{\mathrm{a}}$ & $0.001^{\mathrm{a}}$ & $0.009^{\mathrm{a}}$ & \\
\hline \multirow{3}{*}{ Thin to medium hair } & Before & 17.3 & 32.2 & 27.9 & $0.035^{\mathrm{a}}$ \\
\hline & After & 30.7 & 47.3 & 35.9 & \multirow{2}{*}{0.345} \\
\hline & $P$ value & $0.001^{\mathrm{a}}$ & $0.006^{\mathrm{a}}$ & 0.094 & \\
\hline
\end{tabular}

a value $<0.05$ is statistically significant.

Table 4. Dermoscopy Assessment of Hair Thickness

\begin{tabular}{|c|c|c|c|c|c|}
\hline Mean Thickness of Hair Units ( $\mu \mathrm{m})$ & & $\begin{array}{c}\text { Combined } \\
(n=15)\end{array}$ & $\begin{array}{l}\text { Fractional } \\
\quad(n=15)\end{array}$ & $\begin{array}{c}\text { Minoxidil } \\
(n=15)\end{array}$ & $P$ Value \\
\hline \multirow{3}{*}{ Thick hair } & Before & 80 & 85 & 96 & 0.470 \\
\hline & After & 96 & 106 & 98 & \multirow{2}{*}{0.138} \\
\hline & $P$ value & $0.042^{\mathrm{a}}$ & $0.008^{\mathrm{a}}$ & 0.110 & \\
\hline \multirow{3}{*}{ Thin to medium hair } & Before & 34 & 33 & 35 & $0.035^{\mathrm{a}}$ \\
\hline & After & 47 & 43 & 45 & \multirow{2}{*}{0.345} \\
\hline & $P$ value & $0.001^{\mathrm{a}}$ & $0.001^{\mathrm{a}}$ & $0.001^{\mathrm{a}}$ & \\
\hline
\end{tabular}

$P$ value $<0.05$ is statistically significant.

\section{Global Photos Assessment}

Most of the patients (80\%) showed improvement as (57.8\%) slight improvement (20\%) and moderate improvement, and only one patient $(2.2 \%)$ who was in the combined group showed significant improvement. Twenty percent of the patients showed no improvement. None of the patients were noticed to show deterioration.

\section{Patient's Assessment}

Most of the patients (44/45) were satisfied and only one was dissatisfied. There was no significant difference between the 3 groups regarding the patients' assessment. Most of the patients (97.8\%) noticed their improvement as (17.8\%) slight improvement, (40\%) moderate improvement, and (40\%) significant improvement, and only one patient $(2.2 \%)$ did not notice any improvement. None of the patients were noticed to show deterioration (Figure 4).

Nearly half of the patients (44\%) showed no side effects; the rest of the patients complained of mild side effects as erythema (33\%), itching (16\%) and post-inflammatory hyperpigmentation (7\%).

The main complaint in most of the patients was the lack of compliance (62\%) and only $4 \%$ complained of pain.

Nearly half of the patients (45\%) noticed a decrease in the rate of hair fall; some of them reported an increase followed by a decrease in the rate of hair fall (33\%); however, some reported no change in the rate of hair fall (22\%).

Few patients (11\%) began to notice the improvement
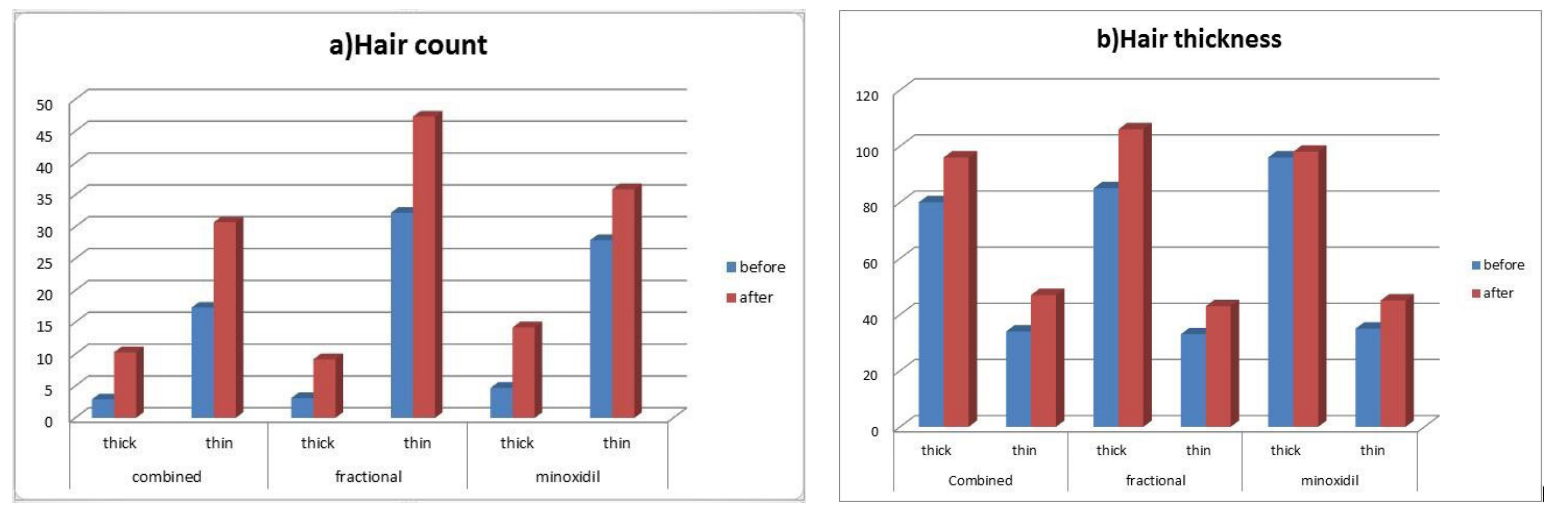

Figure 3. The Graph Chart Showing a Change in (a) the Hair Count (Thin \& Thick) and (b) Hair Thickness After Treatment in Each Group. 
(A)

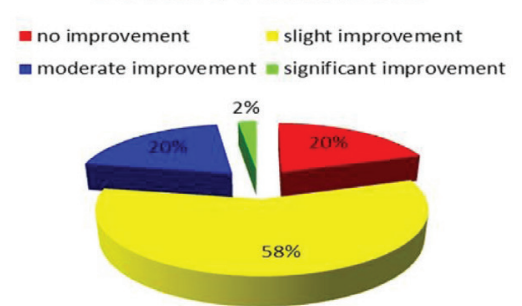

(B) Patients' Assesment

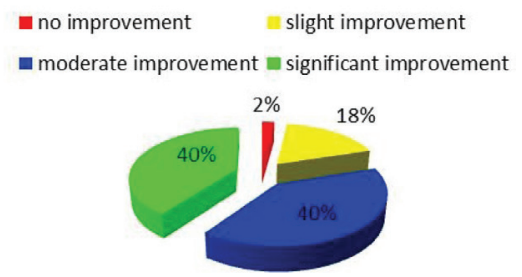

Figure 4. The Pie Chart Showing (A) Doctors' and (B) Patients' Assessment for the Treatment.

after 2 weeks of treatment and some after one month (33\%); however, the majority (56\%) noticed improvement after 2-3 months of treatment.

\section{Discussion}

AGA is a loss of hair which happens as a result of prime susceptivity of hair follicles to androgenic miniaturization. ${ }^{1}$ In AGA, the duration of the anagen phase gradually shortens and that of the telogen phase becomes longer. As the hair length depends on the duration of the anagen phase, the maximum length of the new anagen hair becomes shorter than that of its antecedent, leading to miniaturization and eventually a bald appearance. ${ }^{12}$ Androgenic alopecia is usually found as a moderately stressful condition that decreases body image satisfaction. ${ }^{13}$

In trials to reach satisfactory outcomes, several lines of treatments have been approached. ${ }^{1}$

Topical minoxidil is commonly used in the treatment of AGA; it prolongs the anagen growth phase and gradually develops miniaturized follicles (vellus hairs) into mature terminal hairs. ${ }^{2}$

In the last few years, the use of lasers in treating hair loss has well developed through many means. The paradoxical hair growth noticed after laser hair removal is a clinical example of photo-induced hair growth. A variety of low-level light including the excimer laser, the helium-neon laser, and PUVA (psoralen and ultraviolet type A) has shown success in treating hair loss. ${ }^{3}$ LAD is a new developing science that has grown popular in various medical applications aiming to promote the delivery of medications and thus enhance their therapeutic effect. ${ }^{4}$

Fractional photothermolysis creates a characteristic thermal damage pattern called "microthermal treatment zones (MTZ)" sparing the tissue surrounding each MTZ. It gives "fractional" microscopic thermal columns to the dermis and then creates a healing process. Recently, it has been introduced to treat AGA. ${ }^{5}$ Even though the precise mechanisms of specific fractional lasers on hair regrowth are still not well interpreted, trauma stimulated wound healing mostly has a role. Ito et al found that cells forming the newly developed hair follicles were obtained from the interfollicular epidermis and not the actual hair bulges. This introduced more evidence that appropriate wounding (by ablative fractional lasers) can stimulate hair regrowth. ${ }^{14}$ Hair follicle stem cells supply descendants which travel to the epidermal defect and promote reepithelialization. The process of cutaneous wound healing is augmented markedly during the anagen phase, and more hair follicles regrow after wounding. ${ }^{6}$

Several dermoscopic findings were obtained. The first finding was related to the peripilar sign; in our study, it was found in all the patients before and after treatment. However, there was a significant difference between the 3 groups; it was more present in the fractional group than the other 2 groups after treatment. Rakowska et al reported that in females with AGA (FAGA), the percentage of hair follicles with this finding was significantly higher in the frontal area compared to the occiput. ${ }^{15}$ In another study on Asian people, it was seen in 66\% (33/50) of AGA men and $20 \%(2 / 10)$ of FAGA women. ${ }^{8}$ Other studies noted that the peripilar halo varied from brown to white color. Although there was no specific relationship detected in the occurrence of brown peripilar sign and the stage of hair loss, there was a positive correlation between the white peripilar sign and the progressing stage of AGA besides its duration. ${ }^{11,16}$

Another dermoscopic finding was HDD; it was considered as a hallmark of AGA in other reports. ${ }^{8,11,17,18}$ Similarly, HDD was found in $62.2 \%$ of all the patients and decreased to $40.0 \%$ after treatment. There was no significant difference between the 3 groups regarding hair diversity before and after treatment. However, it was noticed that the percentage decreased after treatment in the combined patient group and the minoxidil group. To the extent of our knowledge, there is no study in the literature about the correlation between MAGA severity and trichoscopic findings according to different disease severity classification; however, Lacarrubba et al underlined miniaturization as being higher in early AGA. ${ }^{19}$

Another feature which was a varying number of yellow spots of different sizes was seen in $100 \%$ of patients before and after treatment and sometimes appeared to be brown. Kibar et al noted brown dots as a marker of severity both in MAGA and FAGA. ${ }^{18}$ It may be assumed that yellow dots in AGA occurred due to the existence of sebaceous lobules, which in histopathology appear large in comparison to the miniaturized follicles. End-organ hypersensitivity to circulating androgens causes the 
sebaceous glands to remain active and produce sebum even after advanced hair follicle miniaturization, which creates intraepidermal sebum lagoons. ${ }^{10}$ The existence of yellow dots was sorted as one of the major criteria in the diagnosis of FAGA and was used to distinguish it from chronic telogen effluvium. ${ }^{14}$ Its presence was also associated with AGA in other studies but with a much lesser percentage. ${ }^{11,15}$

Furthermore, several pinpoint white dots were seen insignificantly in all patients before and after treatment, which were also found in other previous studies. ${ }^{11,18,20}$ It has been found that those white dots could also be seen in healthy individuals with Fitzpatrick skin phototypes IV-VII because they are visible on the contrasting background of the pigmented network $\mathrm{k}^{21}$ and also found in other hair loss disorders as lichen planopilaris and folliculitis decalvans. ${ }^{22}$

Also, arborizing red lines which appear as branching vessels were found in all patients before and after treatment and were also seen in other studies. ${ }^{11,18,23}$ However, they seem to be of no significance.

Another important finding was Multiple follicular hair units (MFHU); hair density is related to the number of hairs emerging from one pilosebaceous unit. ${ }^{9}$ In our study, there was a significant increase in the number of double hair units after treatment in the combined patient group; however, there was no significant increase in the number of triple hair units. Rakowska et al considered the single hair pilosebaceous unit as a minor criterion for AGA, where they noticed that the decreased number of hairs in pilosebaceous units was the main feature differentiating FAGA from most other hair disorders, particularly alopecia areata. ${ }^{15}$ However, in a more recent study, it was shown that MFHUs were statistically significant in MAGA and FAGA when compared with other alopecias and the healthy control group and were also seen to be associated with less severe MAGA and FAGA. ${ }^{18}$

Using the Image Analyzer, hair in the area of treatment was examined as regards its count and thickness. Rakowska et al conducted a study on females and identified 'thin hairs' (below $0.03 \mathrm{~mm}$ ), 'medium-size hairs' (0.03-0.05 mm), and 'thick hairs' (above $0.05 \mathrm{~mm}){ }^{15}$ In our study, hair thickness ranged from $23 \mu \mathrm{m}$ to $115 \mu \mathrm{m}$ and we classified hair less than or equal to $50 \mu \mathrm{m}$ as thin to medium hair and more than $50 \mu \mathrm{m}$ as thick hair.

There was a significant increase in the mean number of hairs after treatment in each group, where it was the most significant in the combined patient group, then in the fractional group, and then in the minoxidil group. However, there was no significant difference in comparing the 3 groups after treatment. There was a significant increase in the count of thick hair after treatment in all the groups, where it was the most significant in the combined patient group and the fractional group and then the minoxidil group. There was a significant increase in the count of thin hair after treatment in the combined patient group and then the fractional group, but not in the minoxidil group. However, there was no significant difference between the 3 groups after treatment.

There was a significant increase in the thickness of thin and thick hair after treatment in the combined patient group and the fractional group, but in the minoxidil group only the thin hair thickness increased; there was no significant difference in comparing between the 3 groups after treatment.

Several clinical trials proved that the ablative fractional laser can improve hair density in patients with AGA. ${ }^{24}$ This can explain the clinical improvement noticed in patients treated with the fractional laser only.

Huang et al assessed the improvement of hair growth in MAGA by a combination of fractional $\mathrm{CO}_{2}$ laser therapy and hair growth factors. In their study, hair density improved in both groups, but the improvement was much more in the combined group than in the growth factor group; the hair shaft diameter also noticeably increased after the treatment. ${ }^{25}$

In conclusion, the fractional $\mathrm{CO}_{2}$ laser in low power can stimulate hair growth. Furthermore, a fractional laser-assisted drug system can be used to supply adjuvant care for patients with MAGA; however, it cannot totally substitute traditional treatment methods. Preferably, the technique could potentially be used in association with standard treatments.

Our study has some recommendations. It is required to increase the duration of treatment and assess convenient maintenance treatment courses. The present observation and treatment periods were to some extent short.

\section{Ethical Considerations}

All procedures performed in this study involving human participants were in accordance with the ethical standards and were approved by the Ethical Committee of National Research Center (NRC) and the Ethical Committee of National Institute of Laser Enhanced Sciences (NILES), Cairo University.

\section{Conflict of Interests}

The authors declare no conflict of interest.

\section{References}

1. McElwee KJ, Shapiro JS. Promising therapies for treating and/or preventing androgenic alopecia. Skin Therapy Lett. 2012;17(6):1-4.

2. Gugle AS, Jadhav VM, Kote R, Deshmukh MD, Dalvi AV. Comparative Study of efficacy of topical Minoxidil 5\% and combination of topical Minoxidil 5\%, topical Azelaic Acid $1.5 \%$ and topical Tretinoin $0.01 \%$ on the Basis of dermoscopic analysis in Androgenetic Alopecia. MVP J Med Sci. 2015;2(2):90-99. doi: 10.15306/mvpjms/2015/ v2i2/78295.

3. Lee GY, Lee SJ, Kim WS. The effect of a $1550 \mathrm{~nm}$ fractional erbium-glass laser in female pattern hair loss. J Eur Acad Dermatol Venereol. 2011;25(12):1450-4. doi: 
10.1111/j.1468-3083.2011.04183.x.

4. Haedersdal M, Erlendsson AM, Paasch U, Anderson RR. Translational medicine in the field of ablative fractional laser (AFXL)-assisted drug delivery: A critical review from basics to current clinical status. J Am Acad Dermatol. 2016;74(5):981-1004. doi: 10.1016/j.jaad.2015.12.008.

5. Yoo KH, Kim MN, Kim BJ, Kim CW. Treatment of alopecia areata with fractional photothermolysis laser. Int J Dermatol. 2010;49(7):845-7. doi: 10.1111/j.13654632.2009.04230.x.

6. Bae JM, Jung HM, Goo B, Park YM. Hair regrowth through wound healing process after ablative fractional laser treatment in a murine model. Lasers Surg Med. 2015;47(5):433-40. doi: 10.1002/1sm.22358.

7. Norwood OT. Male pattern baldness: classification and incidence. South Med J. 1975;68(11):1359-65. doi: 10.1097/00007611-197511000-00009.

8. Inui S, Nakajima T, Itami S. Scalp dermoscopy of androgenetic alopecia in Asian people. J Dermatol. 2009;36(2):82-5. doi: 10.1111/j.1346-8138.2009.00593.x.

9. Leroy T, van Neste D. Contrast enhanced phototrichogram pinpoints scalp hair changes in androgen sensitive areas of male androgenetic alopecia. Skin Res Technol. 2002;8(2):106-11. doi: 10.1034/j.1600-0846.2002.00329.x.

10. Eudy G, Solomon AR. The histopathology of noncicatricial alopecia. Semin Cutan Med Surg. 2006;25(1):35-40. doi: 10.1016/j.sder.2006.01.005.

11. Hu R, Xu F, Han Y, Sheng Y, Qi S, Miao Y, et al. Trichoscopic findings of androgenetic alopecia and their association with disease severity. J Dermatol. 2015;42(6):602-7. doi: 10.1111/1346-8138.12857.

12. Piérard-Franchimont C, Piérard GE. Teloptosis, a turning point in hair shedding biorhythms. Dermatology. 2001;203(2):115-7. doi: 10.1159/000051723.

13. Cash TF. The psychosocial consequences of androgenetic alopecia: a review of the research literature. $\mathrm{Br} \mathrm{J}$ Dermatol. 1999;141(3):398-405. doi: 10.1046/j.13652133.1999.03030.x.

14. Ito M, Yang Z, Andl T, Cui C, Kim N, Millar SE, et al. Wnt-dependent de novo hair follicle regeneration in adult mouse skin after wounding. Nature. 2007;447(7142):31620. doi: 10.1038/nature05766.

15. Rakowska A, Slowinska M, Kowalska-Oledzka E, Olszewska
M, Rudnicka L. Dermoscopy in female androgenic alopecia: method standardization and diagnostic criteria. Int J Trichology. 2009;1(2):123-30. doi: 10.4103/09747753.58555

16. Zhang X, Caulloo S, Zhao Y, Zhang B, Cai Z, Yang J. Female pattern hair loss: clinico-laboratory findings and trichoscopy depending on disease severity. Int J Trichology. 2012;4(1):23-8. doi: 10.4103/0974-7753.96082.

17. Inui S. Trichoscopy for common hair loss diseases: algorithmic method for diagnosis. J Dermatol. 2011;38(1):71-5. doi: 10.1111/j.1346-8138.2010.01119.x.

18. Kibar M, Aktan S, Bilgin M. Scalp dermatoscopic findings in androgenetic alopecia and their relations with disease severity. Ann Dermatol. 2014;26(4):478-84. doi: 10.5021/ ad.2014.26.4.478.

19. Lacarrubba F, Dall'Oglio F, Rita Nasca M, Micali G. Videodermatoscopy enhances diagnostic capability in some forms of hair loss. Am J Clin Dermatol. 2004;5(3):2058. doi: 10.2165/00128071-200405030-00009.

20. Ross EK, Vincenzi C, Tosti A. Videodermoscopy in the evaluation of hair and scalp disorders. J Am Acad Dermatol. 2006;55(5):799-806. doi: 10.1016/j.jaad.2006.04.058.

21. Sewell LD, Elston DM, Dorion RP. "Anisotrichosis": a novel term to describe pattern alopecia. J Am Acad Dermatol. 2007;56(5):856. doi: 10.1016/j.jaad.2007.01.020.

22. Rudnicka L, Olszewska M, Rakowska A, Slowinska M, Walecka I, Borkowska B, et al. Hair follicle openings: dots. In: Rudnicka L, Olszewska M, Rakowska A, editors. Atlas of Trichoscopy: Dermoscopy in Hair and Scalp Disease. London: Springer; 2012. P. 47-71. doi: 10.1007/978-1-44714486-1.

23. Karadağ Köse Ö, Güleç AT. Clinical evaluation of alopecias using a handheld dermatoscope. J Am Acad Dermatol. 2012;67(2):206-14. doi: 10.1016/j.jaad.2011.08.019.

24. Perper M, Aldahan AS, Fayne RA, Emerson CP, Nouri K. Efficacy of fractional lasers in treating alopecia: a literature review. Lasers Med Sci. 2017;32(8):1919-1925. doi: 10.1007/ s10103-017-2306-7.

25. Huang Y, Zhuo F, Li L. Enhancing hair growth in male androgenetic alopecia by a combination of fractional $\mathrm{CO}_{2}$ laser therapy and hair growth factors. Lasers Med Sci. 2017; 32(8):1711-1718. doi: 10.1007/s10103-017-2232-8. 